\title{
Dynamic Covalent Dextran Hydrogels as Injectable, Self-adjuvating Peptide Vaccine Depots
}

\author{
Bowen Fan ${ }^{1, \#}$, Diana Torres García ${ }^{2, \#}$, Marziye Salehi1 ${ }^{1,2}$, Sander I. van Kasteren²,*, Rienk \\ Eelkema $^{1, *}$ \\ ${ }^{1}$ Department of Chemical Engineering, Delft University of Technology \\ Van der Maasweg 9, 2629 HZ Delft, The Netherlands
}

${ }^{2}$ Leiden Institute of Chemistry and Institute of Chemical Immunology, Division of Bio-Organic Synthesis, Leiden University, Gorlaeus Laboratory, Einsteinweg 55, 2333 CC, Leiden, The

Netherlands

\# equal contributions

* corresponding authors; e-mail: r.eelkema@tudelft.nl, s.i.van.kasteren@chem.leidenuniv.nl

Keywords: dynamic covalent hydrogels, vaccines, injectable gel, dextran, peptide antigens.

\begin{abstract}
Dextran-based hydrogels are promising therapeutic materials for drug delivery, tissue regeneration devices, and cell therapy vectors, due to their high biocompatibility, along with their ability to protect and release active therapeutic agents. This report describes the synthesis, characterization and application of a new dynamic covalent dextran hydrogel as an injectable depot for peptide vaccines. Dynamic covalent crosslinks based on double Michael addition of thiols to alkynones impart the dextran hydrogel with shear-thinning and self-healing capabilities, enabling hydrogel injection. These injectable, non-toxic hydrogels show adjuvant potential and have predictable sub-millimolar loading and release of the peptide antigen SIINFEKL, which after its release is able to activate T-cells, demonstrating that the hydrogels deliver peptides without modifying their immunogenicity. This work demonstrates the potential of dynamic covalent dextran hydrogels as a sustained-release material for delivery of peptide vaccines.
\end{abstract}


The success of vaccines is strongly dependent on the kinetics of antigen exposure and the subsequent cellular and humoral immune responses induced. ${ }^{[1]}$ Sustained antigenic exposure within tightly controlled release conditions is therefore sought after to prompt a durable and protective immune response. ${ }^{[1-3]}$ Sustained-release technologies such as nanoparticles, cationic lipids/liposomes, polysaccharides, and poly (lactic-co-glycolic acid) (PLGA) particles can provide these essential spatial and temporal interactions. ${ }^{[3-5]}$ Nevertheless, these systems must overcome some challenges such as tedious synthesis procedures, low drug loading capacity, or inability to deliver cargo. As for the cargo, the loaded antigens could face degradation and immunogenicity loss induced by changes in $\mathrm{pH}$, temperature, oxidation/reduction reactions, or other chemical modifications. ${ }^{[6]}$ Thus, there is an urgent need for delivery systems capable of releasing unmodified antigens. In this regard, an excellent alternative for such systems can be found in hydrogels, as they can possess high biocompatibility as well as high loading capacity. ${ }^{[7,8]}$ Their hydrophilic nature enables the absorption of large amounts of fluid and bioactive cargo.

The use of hydrophilic polymer scaffolds ensures a low cellular and protein adherence to the gel interface, making them biocompatible. ${ }^{[6,9]}$ Viscoelastic properties and in vivo degradation characteristics can be tuned through molecular design. ${ }^{[10,11]}$ The chemical and mechanical properties of hydrogels determine the release kinetics of the cargo, which can be controlled by altering the polymer structure, the density, and type of crosslinker forming the hydrogel, as well as its degradation kinetics. ${ }^{[12-14]}$ Hydrogels can act as depots for the sustained release of antigens. [15-17] These depot gels can be introduced in the body by grafting to the skin, surgical implantation, and through injection. Injection requires shear-thinning and self-healing properties that are not observed for permanently crosslinked polymer hydrogels but can be introduced through the use of reversible crosslinks. In this context, Appel et al. described a cellulose-derived hydrogel containing hydrophobic non-covalent crosslinks, for the sustained release of model protein antigens. ${ }^{[15]}$

In an effort to develop injectable hydrogel antigen depots that do not use hydrophobic interactions, we were interested to evaluate the use of dextran polymers crosslinked through dynamic covalent bonds, for the release of peptide antigens. Peptide vaccines have been at the forefront in the recent spate of 'molecularly defined' anti-cancer vaccines. ${ }^{[18,19]}$ However, to date they have precluded hydrogel-based delivery; with only large protein antigens having been 
delivered in injectable hydrogels. The small size and poor solubility of many antigenic peptides mean that delivering these agents presents additional restraints on the carrier materials.

Here we report the synthesis, characterization, and mechanical properties of an injectable dextran hydrogel containing dynamic covalent crosslinks, and evaluation of prolonged T-cell activation by the release of peptide antigens. The biocompatibility of dextran has been previously reported making this polymeric hydrogel a very suitable system for the controlled release of antigens. ${ }^{[20-}$ ${ }^{22]}$ As a dynamic covalent crosslink, we use the double Michael addition of thiols to alkynones, affording a reversible dithiane link that will slowly degrade in the presence of small-molecule thiols. We demonstrate the feasibility of this novel dextran hydrogel as a delivery system, by loading it with the SIINFEKL peptide, a minimal CD8-restricted T-cell epitope peptide. Moreover, this peptide was successfully released without losing its immunogenicity and it was taken up and processed by dendritic cells, resulting in its efficient presentation in the context of MHC class I molecules, and the subsequent antigen-specific T-cell activation.

We have recently reported a polymer hydrogel that is crosslinked through thiol-alkynone Michael addition dynamic covalent chemistry. ${ }^{[23]}$ The gels were made from tetra-thiol PEG star polymers that react with a small molecule alkynone to form a $\beta$-dithiane carbonyl dynamic crosslink. ${ }^{[24-26]}$ Resulting from the presence of this dynamic covalent crosslink, these polymer gels are shear thinning and self-heal after a stress is removed. Because of that property, the gels can be injected using a syringe, where they form stable gel particles immediately upon exiting the needle. Combining injectability with high water content and crosslinks that show dynamic behavior under physiological conditions make these materials interesting candidates for evaluation as injectable antigen depot vehicles for vaccination.

The original research employed a tetrathiol PEG star polymer as the polymer backbone of the gel. Such star polymers have limited availability and are difficult to functionalize further. Moreover, the free thiol groups may form disulfides through oxidation, leading to undesired crosslinking. Building on this work, we opted to develop a dextran-based polymer with side chain grafted lipoic acid as a masked dithiol. Reduction of the lipoic acid disulfide leads to the formation of two thiol functionalities, which mostly revert to the ring closed disulfide upon oxidation, thereby avoiding undesired crosslinking. Using a graft copolymer instead of an endfunctionalized star polymer allows control over the crosslink-to-polymer ratio. After reduction, the free thiols on the lipoic acid-functionalized dextran $\mathbf{P}$ can react with alkynone $\mathbf{A}$ to form a 
polymer network crosslinked with dynamic covalent double Michael addition products (Figure $1)$.

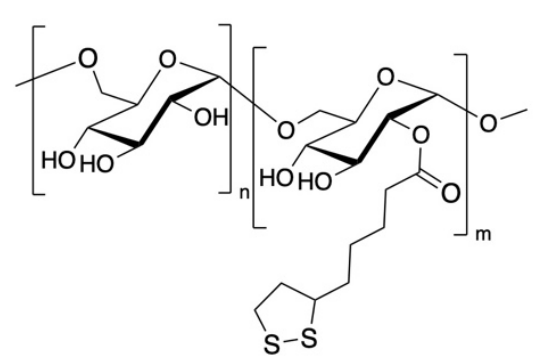

dextran-lipoic acid Dex-P

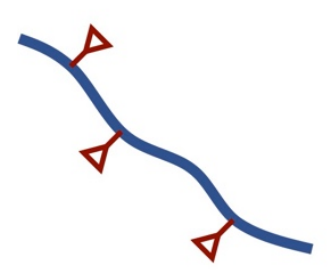

dextran-lipoic acid $\mathbf{P}$

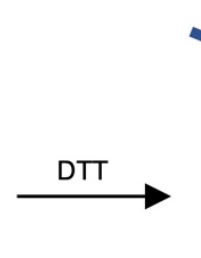

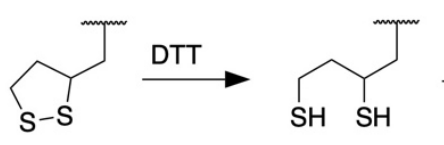

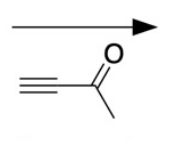

alkynone A

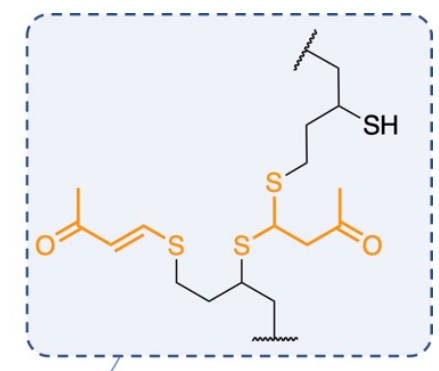

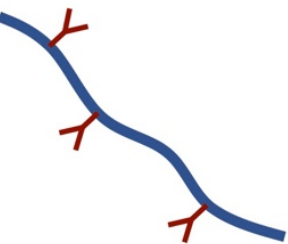

reduced $\mathbf{P}$

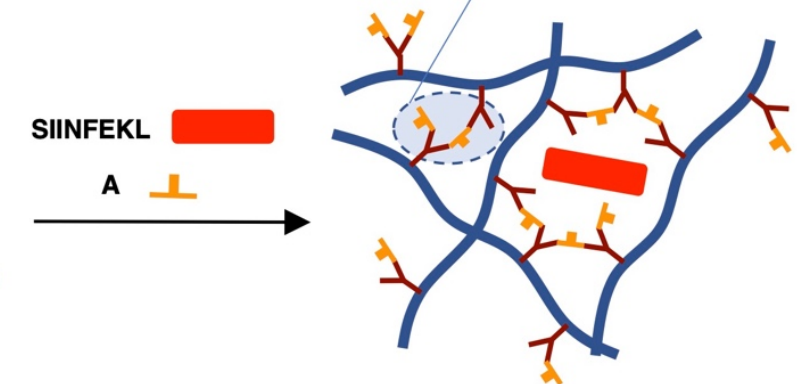

Dex P-A gel with loaded antigen

Figure 1. General concept and materials. Thiols formed by reduction of lipoic acid side chains on dextran polymer Dex-P react with alkynone $\mathbf{A}$, to form the single and double Michael adducts. A-crosslinked polymer gels (Dex P-A) can be loaded with peptide antigens such as SIINFEKL and used for T-cell activation.

To make the hydrogel-vaccine, we first synthesized lipoic acid-functionalized dextran (Dex-Px-y, with $\mathrm{x}$ indicating the dextran molecular weight, and $\mathrm{y}$ indicating the degree of functionalization with lipoic acid). We synthesized several versions of Dex-P using two methods: dextran was either reacted with lipoic acid anhydride catalyzed by dimethylaminopyridine (DMAP), or with lipoic acid catalyzed by DPTS (the 4-toluenesulfonate salt of DMAP). Starting from dextran with $\mathrm{Mw}=20 \mathrm{kDa}, 70 \mathrm{kDa}$, and $500 \mathrm{kDa}$, we synthesized Dex-P polymers with degrees of substitution between 3.1 and 10.5 (Table 1). Dex-P polymers with a high degree of substitution were not sufficiently soluble in aqueous solvent, and were not investigated further. We next determined whether the polymers could form gels upon reaction with alkynone $\mathbf{A}$ in sodium phosphate buffer (100 mM phosphate, $\mathrm{pH}=8.2$, "PB8.2"). For the dithiothreitol (DTT)-mediated reduction of native lipoic acid, we determined that around $67 \%$ disulfide is reduced in 10 minutes, using 1 eq. DTT in PB8.2 (Figure S6). Based on this result, we treated a polymer solution for $10 \mathrm{~min}$. 
with DTT (1 eq. with respect to the dithiolane ring content of the polymer) to reduce the lipoic acid sidechain disulfides. After DTT reduction, we added A (1 eq. to DTT), and the mixtures were left to react for 4 hours at room temperature to allow crosslink formation. Of the soluble Dex-P polymers, only the $70 \mathrm{kDa}$ dextran with $\mathrm{DS}=4.2$ (P70-4.2), made using the anhydride method, showed gelation. This polymer formed a turbid hydrogel after activation with DTT and subsequent reaction with A (10 wt\% polymer in PB8.2) (Figure 2a). P70-4.2-d synthesized by the DPTS catalysis method does not form a gel. This effect may be caused by different substitution patterns of dextran depending on the use of the anhydride method or DPTS. For P70-4.2, the ${ }^{1} \mathrm{H}$ NMR spectrum shows that lipoic acid esterification takes place mostly at the $\mathrm{C} 2$ hydroxyls of dextran (Figure S2). For P70-4.2-d, however, a new peak at $5.27 \mathrm{ppm}$ in the ${ }^{1} \mathrm{H}$ NMR spectrum suggests substantial esterification at both C3 and C2 hydroxyls (Figure S5). ${ }^{[27]}$

All other polymers gave liquid solutions after incubation with $\mathbf{A}$. The solubility and gelation results suggest that there is a fine balance between having enough lipoic acid groups per polymer chain to allow sufficient crosslink formation and having too many hydrophobic lipoic acid groups hindering solubility.

We investigated the process of hydrogel formation and the mechanical properties of the formed gels by rheological measurements. After reacting for 10 minutes with DTT, we added A to the solution of activated P70-4.2, and the mixture was transferred on the rheometer. A rheological time sweep shows a fast gelation, indicated by the crossover of storage modulus $\left(G^{\prime}\right)$ and loss modulus $\left(G^{\prime \prime}\right)$ after 5 minutes. After $6 \mathrm{~h}$ of reaction, $G^{\prime}$ approached an equilibrium value $\left(G^{\prime}=\right.$ $5.0 \mathrm{~Pa}$ and $\tan \delta\left(G^{\prime \prime} / G^{\prime}\right)=0.34$ ) (Figure S7a). A frequency sweep demonstrated that the hydrogel maintains a solid-like state in the range of 100 to $0.01 \mathrm{rad} \mathrm{s}^{-1}$ (Figure 2b).

Table 1. Synthesis, solubility, and gelation of Dex-P with varying molecular weight and degree of substitution.

\begin{tabular}{cccccc}
\hline Dex-P & $\begin{array}{c}\text { dextran } \\
\text { Mw (kDa) }\end{array}$ & $\begin{array}{c}\text { molar ratio of } \\
\text { LA to AHG }^{\text {a }}\end{array}$ & $\begin{array}{c}\text { degree of } \\
\text { substitution }^{\text {b }}\end{array}$ & P solubility $^{\mathrm{c}}$ & gelation $^{\text {e }}$ \\
\hline P20-6.4 & 20 & 0.5 & 6.4 & soluble & liquid $^{\text {d }}$ \\
P70-3.1 & 70 & 0.3 & 3.1 & soluble & liquid $^{\text {d }}$ \\
P70-4.2 & $\mathbf{7 0}$ & $\mathbf{0 . 5}$ & $\mathbf{4 . 2}$ & soluble & gel $^{\text {P70-5.9 }}$ \\
\hline
\end{tabular}




\begin{tabular}{cccccc}
\hline $\mathrm{P} 70-4.2-\mathrm{d}^{\mathrm{f}}$ & 70 & 0.4 & 4.2 & soluble & liquid $^{\mathrm{d}}$ \\
$\mathrm{P} 70-6.8-\mathrm{d}^{\mathrm{f}}$ & 70 & 0.6 & 6.8 & low solubility & - \\
$\mathrm{P} 70-10.5-\mathrm{d}^{\mathrm{f}}$ & 70 & 0.7 & 10.5 & low solubility & - \\
$\mathrm{P} 500-4.8$ & 500 & 0.5 & 4.8 & low solubility & - \\
\hline
\end{tabular}

${ }^{a}$ Molar feeding ratio of lipoic acid or lipoic acid anhydride to AHG of dextran during the synthesis of Dex P-A. ${ }^{b}$ Degree of substitution is defined as the number of attached lipoic acid units per $100 \mathrm{AHG}$ units of dextran and calculated by ${ }^{1} \mathrm{H}$ NMR according to the protons of the attached lipoic acid group at $3.10 \mathrm{ppm}$ and the dextran glucosidic protons at 4.85 and $5.19 \mathrm{ppm} .{ }^{\mathrm{c}}$ In PB8.2 (20 mg in $180 \mu \mathrm{L}$ buffer). Low solubility means that even stirred overnight or processed with ultra-sonication for $1 \mathrm{~h}$ does not lead to complete solubilization. ${ }^{\mathrm{d}}$ The sample still shows flow after 1 day reaction time, checked by the vial-inversion method. ${ }^{\mathrm{e}}$ Hydrogel formation (10 wt \% polymer in PB8.2) was checked by the vial-inversion method. Gel formation means the sample shows no flow within 1 minute after inversion. ${ }^{\mathrm{f}}$ Synthesized from dextran-70k and lipoic acid catalyzed by DPTS.

The critical strain $(\gamma)$ needed to induce a gel-sol transition was determined by a strain sweep from 1 to $1200 \%$ (Figure S7c), showing a crossover point of $G$ ' and $G$ " at around $1000 \%$. We then examined the ability to self-heal by a continuous step-strain sweep using a cyclic $1 \%$ to $1200 \%$ strain program (Figure 2c). Upon applying a 1200\% strain to the hydrogel, $G$ ” becomes higher than $G^{\prime}$, which means that the hydrogel turns to a fluid state. When the applied strain turned to $1 \%, G$ ' recovered back immediately to around $3 \mathrm{~Pa}$ and $\tan \delta<1$, suggesting a rapid self-healing of the hydrogel. At a second strain cycle, the hydrogel again showed self-healing after fluidization and $G^{\prime}$ again subsequently recovered to the initial value. In addition, we demonstrated the self-healing ability of the hydrogel by a macroscopic self-healing test (Figure $2 \mathrm{e})$. Two cube-shaped Dex P-A hydrogels $(4 \times 10 \times 10 \mathrm{~mm})$ were colored yellow and red by fluorescein and rhodamine B dyes, respectively. The two hydrogels were then weakly pressed together and kept in a humid atmosphere to allow self-healing. After 10 minutes, the two hydrogels had connected and the resulting gel could be lifted using a tweezer, without the newly formed connection failing. The crack between the two gels subsequently disappeared, enabling observable diffusion of dyes across the interface (Figure 2d). We demonstrated injection of the 
hydrogel by extruding a colored Dex P-A hydrogel through a $20 \mathrm{G}$ syringe needle (Figure 2e). After exiting the needle, the gel healed immediately and could be drawn as an ink.

a)

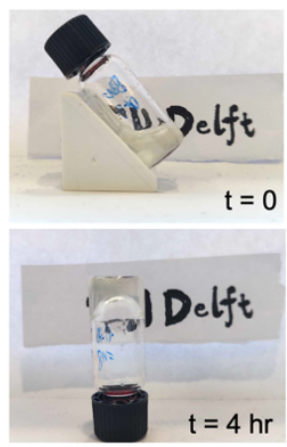

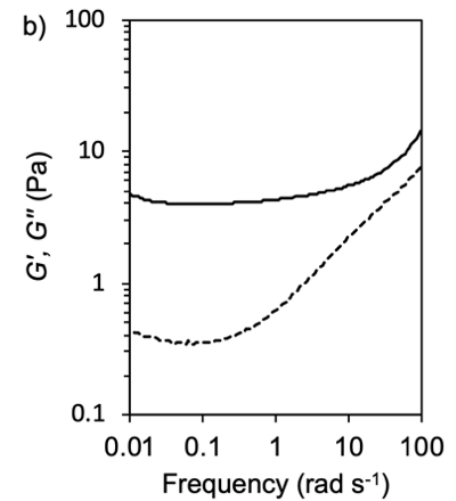

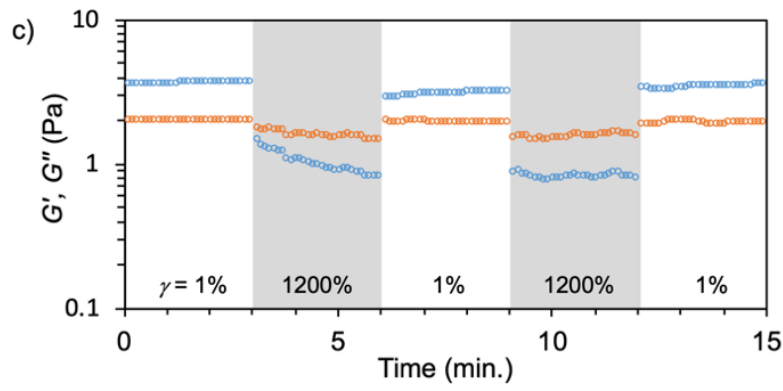

d)

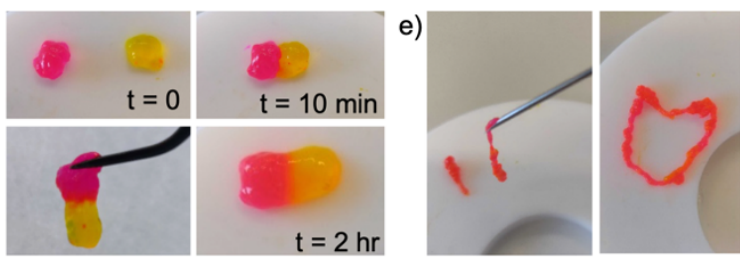

Figure 2. Dex P-A hydrogel formation and its mechanical properties, self-healing, and injectability. a) Hydrogel formation by mixing P70-4.2, DTT, and alkynone A PB8.2 solutions sequentially: after 10 minutes of mixing P70-4.2 and DTT solutions, $\mathbf{A}$ in PB8.2 was added into the mixture, initiating the gelling process (top); after $4 \mathrm{~h}$ reaction, a slightly turbid hydrogel formed, that could hold its own weight when inverted (bottom). b) Rheological frequency sweep of the hydrogel $6 \mathrm{~h}$ after mixing, showing that $G^{\prime}$ (solid line) is higher than $G^{\prime \prime}$ (dashed line) over the entire frequency range ( $\operatorname{strain}(\gamma)=0.5 \%$, frequency $=100-0.01 \mathrm{rad} \mathrm{s}^{-1}, 25^{\circ} \mathrm{C}$ ). c) Continuous step strain measurement of the hydrogel, strain is switched from $1 \%$ to $1200 \%$ for two cycles. d) Macroscopic self-healing of the hydrogel. Two cube-shaped gels $(4 \times 10 \times 10 \mathrm{~mm})$ were colored red and yellow using rhodamine B and fluorescein, respectively (top left). The two gels were pressed together and found to have connected after $10 \mathrm{~min}$ (top right). The rejoined gel can be lifted using a tweezer (bottom left). After $2 \mathrm{~h}$, the interface between the two gels had disappeared 
and the dyes could diffuse over the interface (zoom, bottom right). e) A $0.6 \pm 0.2 \mathrm{~mm}$ stripshaped hydrogel formed after hand-pressed extrusion through a $20 \mathrm{G}$ syringe needle (left). Rhodamine B was added to the gels for visualization. The extruded structures could hold their shape over extended periods (right).

We next determined the cytotoxicity of the Dex P-A hydrogel to immune cells. For this, the dendritic cell line D1 ${ }^{[28]}$ was cultured on Dex P-A hydrogel and the viability was evaluated through an MTT assay. This assay measures the reduction of MTT into formazan by mitochondrial succinate dehydrogenase. ${ }^{[29]}$ The concentration of formazan is directly proportional to the number of live cells; therefore, possible detrimental intracellular effects on metabolic activity associated with hydrogel toxicity will influence the outcome. The D1 cells were cultured on Dex P-A for 24 and 48 h prior to washing off the hydrogel with PBS to remove excess free reagent and any soluble by-product. Under these conditions, $82 \pm 11 \%$ and $79 \pm 9 \%$ of D1 cells survived after $24 \mathrm{~h}$ and $48 \mathrm{~h}$ of incubation, respectively (Figure 3). If the washing step was omitted, viability decreased by approximately $30 \%$ ( $<<0.05$ compared with 24 and $48 \mathrm{~h}$ incubation times), suggesting residual soluble components of the gel formation being toxic to the cells.

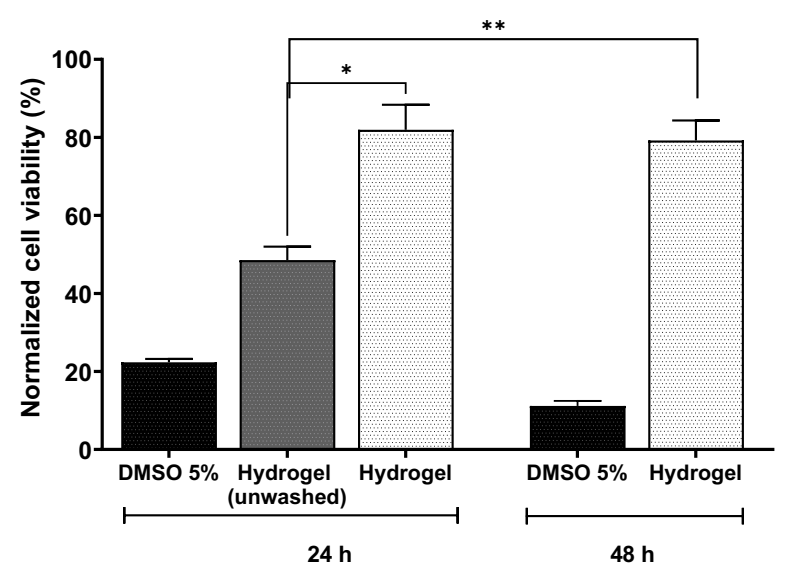

Figure 3. Cell viability on the Dex P-A hydrogel. MTT assay in D1 cells cultured on Dex P-A hydrogel incubated for 24 and $48 \mathrm{~h}$ at $37^{\circ} \mathrm{C}, 5 \% \mathrm{CO}_{2}$, and $95 \%$ humidity. Error bars represent the standard error of the mean. Data correspond to 3 independent experiments. The asterisks indicate the difference between the unwashed Dex P-A hydrogel and the other hydrogels. ${ }^{*} p=0.01{ }^{* *} p=$ 0.007 . 
To assess the potential of these hydrogels as a peptide vaccine delivery systems, we evaluated the release of the commonly used minimal CD8-restricted T-cell epitope peptide SIINFEKL ${ }^{[30]}$ from the Dex P-A hydrogel. For this, Dex P-A hydrogels were loaded with three different concentrations of SIINFEKL $(1,10$, and $100 \mu \mathrm{M})$. The release of SIINFEKL over time was detected through an (in vitro) T-cell activation assay, where the amount of peptide is quantified using the T-cell clone B3Z that carries a LacZ gene under the NFAT promoter, that allows it to produce beta-galactosidase in response to the peptide loaded on MHC-I in a concentration dependent manner. ${ }^{[31]}$ This in turn can be quantified using the conversion of the luminogenic substrate chlorophenol red- $\beta$-D-galactopyranoside (CPRG). Figure 4 shows that the release rate of the SIINFEKL from the Dex P-A hydrogels is influenced by the concentration of peptide loaded in the hydrogels. In this regard, the maximum cumulative \% of SIINFEKL release at 48 hours is 15,27 , and $37 \%$ for the Dex P-A hydrogels loaded with 1,10 , and $100 \mu \mathrm{M}$ of SIINFEKL, respectively (Figure 4a-c). An experiment at the higher $1000 \mu \mathrm{M}$ concentration (Figure S8) confirmed this trend.

Based on the above, we compared the release profiles of SIINFEKL at $48 \mathrm{~h}$ from Dex P-A hydrogels loaded with $1,10,100$, and $1000 \mu \mathrm{M}$ concentrations (Figure 4d). The total amount of SIINFEKL released is related to the loading concentration by a power law with a 0.23 exponent. Combined, these results suggest that the loaded peptide is entrapped within the mesh network of the Dex P-A hydrogel and released either as degradation of the hydrogel matrix occurs, or though diffusion from the intact matrix. The time-dependent release data (Figure 4 a-c) does not show zeroth or first order kinetics, and has only a partial fit to the Higuchi equation ${ }^{[32]}$, suggesting that release is not merely governed by Fickian diffusion from the hydrogel matrix. A time lag observed in all release profiles also suggests that changes to the hydrogel network play a role in the release. A fit to the Korsmeyer-Peppas model ${ }^{[33]}$ shows that, after the lag time, the release process is initially largely dominated by Fickian diffusion (exponent $\mathrm{n} \sim 0.5$ ) followed by an increase of $\mathrm{n}$ on longer time scales, suggesting a combination of diffusion and hydrogel erosion. We therefore postulate that both diffusion and degradation play a role in the release, with relative contributions changing over time. Interestingly, only the gel with the extreme 1000 uM loading showed a burst release. No burst phase release of the peptide antigen was observed at the more relevant 1-100 $\mu \mathrm{M}$ loading concentrations. 
The cumulative percentage of SIINFEKL release at $48 \mathrm{~h}$ indicates that significant amounts of SIINFEKL are retained in the hydrogel. We therefore measured the residual T-cell activation capacity of the $48 \mathrm{~h}$ gels. For this purpose, we disrupted the remaining hydrogel mechanically, and incubated the residue at $37{ }^{\circ} \mathrm{C}$ for 20 minutes. After this time, the gel had dissolved completely, confirming its biodegradation potential. We subsequently pulsed D1 cells with the dissolved gels and measured T-cell activation. Interestingly, the Dex P-A hydrogel by itself was also able to activate the B3Z T-cells. This result suggests that the Dex P-A hydrogel in itself has immunostimulatory properties. This could be beneficial for increasing antigen-presenting cell (APC) activation, as has been reported in previous studies with other hydrogels and proteindextran conjugates. ${ }^{[2,34]}$ This T-cell activation induced by non-loaded hydrogels (Figure S8) hints towards a potential local inflammatory niche formation capacity, that could enhance immune responses. However, the impact of their specific niche properties, its persistence, and relevance to the immune response induced must be explored in future in vivo assays.
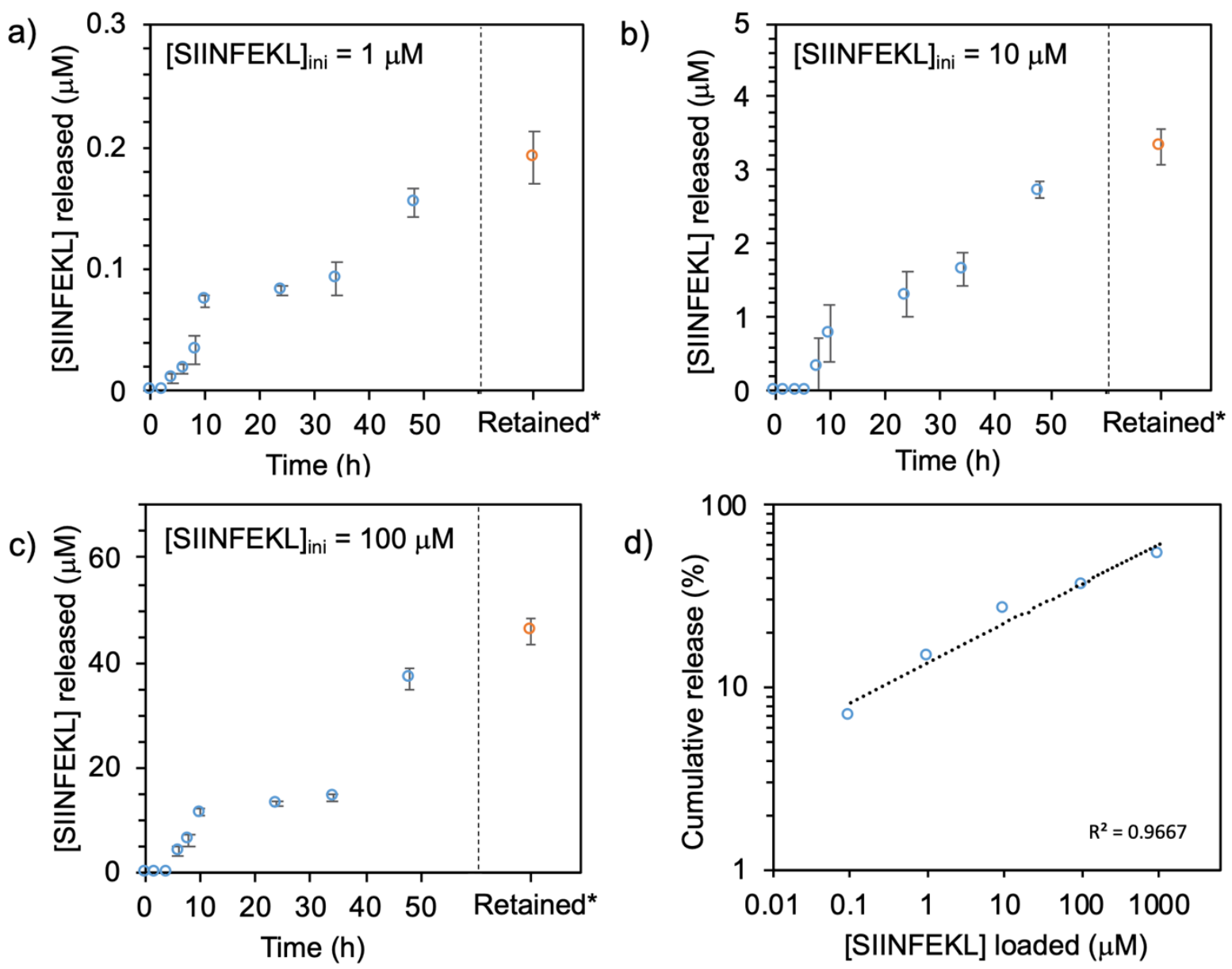
Figure 4. SIINFEKL release from the hydrogel. SIINFEKL release from Dex P-A hydrogel over a $48 \mathrm{~h}$ period. D1 cells were pulsed for $3 \mathrm{~h}$ with supernatant released from Dex P-A hydrogel loaded with $1 \mu \mathrm{M}$ (a), $10 \mu \mathrm{M}$ (b), $100 \mu \mathrm{M}$ (c) of SIINFEKL, and then co-cultured with B3Z cells to analyze their activation. *Retained indicates SIINFEKL retained in the remaining hydrogel after 48 h. d) SIINFEKL release profile at 48 h of Dex P-A hydrogels loaded with 1, 10,100 and $1000 \mu \mathrm{M}$ concentrations, plotted on a double logarithmic scale. The data fits a power law with a $0.23 \pm 0.02$ slope, describing the relation between loaded and released peptide. Error bars represent the SD. Data correspond to 4 independent experiments $(n=2$ replicates per experiment).

In this work, we show how dextran derived polymer gels can act as injectable depots for sustained release of vaccines. We developed a dextran polymer that is crosslinked using a dynamic covalent double Michael addition of thiols to alkynones. Using a masked thiol prevents undesired oxidative thiol crosslinking of the matrix polymer. The obtained hydrogels are shear thinning and self-healing, and can be injected through a $20 \mathrm{G}$ needle, forming stable gel particles immediately after extrusion. These hydrogels show acceptable viability of dendritic cells, suggesting that they are compatible for interacting with the immune system. In vitro tests show slow, sustained release of loaded SIINFEKL minimal epitope, as measured by T-cell activation essays. The rate of T-cell activation depends on the concentration of loaded antigen. This, combined with the observed self-adjuvating properties of the hydrogels, suggest that they could find application as injectable depots for slow and prolonged release of vaccines, which could be used to achieve augmented vaccination response.

\section{Experimental Section}

NMR spectra were recorded on an Agilent-400 MR DD2 (399.7 MHz for ${ }^{1} \mathrm{H}$ and $100.5 \mathrm{MHz}$ for ${ }^{13} \mathrm{C}$ ) at $298 \mathrm{~K}$. The rheological measurements were performed using a rheometer (AR G2, TA instruments) equipped with a steel plate-and-plate geometry of $40 \mathrm{~mm}$ in diameter and equipped with a hexadecane trap. Dithiothreitol, lipoic acid (LA) and 3-butyn-2-one were purchased from Fluorochem ltd. Dextran-500k $\left(\mathrm{M}_{\mathrm{n}}=500 \mathrm{kDa}\right)$ and dextran-20k $\left(\mathrm{M}_{\mathrm{n}}=20 \mathrm{kDa}\right)$ were purchased from Alfa Aesar. Dextran-70k $\left(\mathrm{M}_{\mathrm{n}}=70 \mathrm{kDa}\right), \mathrm{N}, \mathrm{N}^{\prime}$-dicyclohexylcarbodiimide (DCC), p- 
toluenesulfonic acid monohydrate and 4-(dimethylamino) pyridine (DMAP) were purchased from Sigma Aldrich. 4-(Dimethylamino)pyridinium 4-toluenesulfonate (DPTS) was synthesized according to previous literature. ${ }^{[35]}$ The technical solvents were purchased from VWR and the reagent grade solvents were purchased from Sigma Aldrich.

Cell lines and culture: D1 and B3Z (OVA257-264-specific, H2kb-restricted CTL hybridome) cell lines were cultured in IMDM (Iscove's Modified Dulbecco's Medium) complete medium, supplemented with $10 \%$ heated-inactivated fetal calf serum (FCS), antibiotics $\left(100 \mu \mathrm{gmL}^{-1}\right.$ streptomycin and $100 \mathrm{IUmL}^{-1}$ penicilin), $2 \mathrm{mM}$ glutamine (glutamax), and $50 \mathrm{mM} 2-\beta$ mercaptoethanol. In addition, the D1 cell-medium was supplemented with $30 \%$ rGM-CSF mouse (10-20 ngmL $\left.{ }^{-1}\right)$. This growth factor was collected and filtered from the supernatant of NIH/3T3 cell-cultures. The cell lines were cultured at $37^{\circ} \mathrm{C}$ with $95 \%$ relative humidity and $5 \% \mathrm{CO}_{2}$ atmosphere. Cultured cells were harvested by PBS-EDTA and washed two times with medium.

Synthesis of Lipoic acid anhydride: Lipoid acid anhydride was synthesized based on the method described by previous literature. ${ }^{[36]}$ A mixture of lipoic acid (6.00 g, $\left.29.08 \mathrm{mmol}\right)$ and DCC (3.60 g, $17.45 \mathrm{mmol})$ was stirred in $80 \mathrm{~mL}$ of anhydrous dichloromethane at room temperature under nitrogen atmosphere. After 20 hours, the product mixture was filtered to remove dicyclohexylurea and solvent was removed by rotary evaporation. The product was dried in an oven overnight to obtain a yellow solid (3.59 g, yield: $60 \%$ ). The product was directly used in next step without purification.

Synthesis of lipoic acid-functionalized dextran P1 by esterification with acid anhydride method: Lipoic acid-functionalized dextran (Dex-P) was synthesized based on the method described in the literature. ${ }^{[37]}$ Dextran-70k (1.41 g, containing 8.69 mmol AHG), 4(dimethylamino) pyridine (1.06 g, $8.69 \mathrm{mmol})$ and lipoic acid anhydride $(1.72 \mathrm{~g}, 4.35 \mathrm{mmol}$, ratio of lipoic acid anhydride to anhydroglucosidic rings of dextran (AHG) is 0.5 ) were dissolved to $20 \mathrm{~mL}$ anhydrous DMSO. The mixture was stirred for 48 hours at $50{ }^{\circ} \mathrm{C}$. Then, the product was precipitated in cold ethanol. The precipitation was collected by centrifugation and dissolved in water. The mixture solution was transferred to a dialysis bag $(\mathrm{MWCO}=14 \mathrm{kDa})$ and dialyzed by distilled water for 2 days. The white solid was obtained after freeze-drying ("P70", $0.47 \mathrm{~g}$, yield, 34\%). The degree of substitution (DS, defined as the number of attached lipoic acid rings per 100 AHG unit) is 4.2 determined by ${ }^{1} \mathrm{H}$ NMR (ratio based on the integration of peak at 3.10 ppm and the integration of peaks at 4.85 and 5.19). ${ }^{1} \mathrm{H}$ NMR (399.7 MHz, $\left.\mathrm{D}_{2} \mathrm{O}\right) \delta 5.19,5.05$, 
4.85 (m, dextran anomeric protons), 3.62 (m, hydroxyl of dextran), 3.10 (m, $\left.-\mathrm{SS}-\underline{\mathrm{CH}}_{2}-\mathrm{CH}_{2}-\mathrm{CH}\right)$, 2.37 (m, -SS-CH${ }_{2}-\mathrm{CH}_{2}-\mathrm{CH}$ and $\left.-\underline{\mathrm{CH}}_{2}-\mathrm{COO}\right), 1.88$ (m, -SS-CH${ }_{2}-\underline{C H}_{2}-\mathrm{CH}$ ), 1.63, 1.54, 1.35 (m, $\mathrm{CH}_{2}-\mathrm{CH}_{2}-\mathrm{CH}_{2}-$ dithiolane ring).

Dex-P based on dextran-500k (P500) and Dex-P based on dextran-20k (P20) were synthesized by the same procedure as described above.

P70 with varying degree of substitution (3.1 and 5.9) were obtained by using different molar ratios of lipoic acid anhydrides to AHG of dextran (0.3 and 0.8).

Synthesis of lipoic acid-functionalized dextran P70d by esterification using DPTS as a catalyst system: Dextran-70k (0.981 g, containing $6.05 \mathrm{mmol}$ AHG), lipoic acid (0.5 g, 2.42 mmol, 0.4 eq. to AHG of Dextran), DPTS (0.107 g, $0.363 \mathrm{mmol}, 0.15$ eq. to acid) and DCC (0.75 g, $7.27 \mathrm{mmol})$ were dissolved to $30 \mathrm{~mL}$ anhydrous DMSO. The mixture was stirred for 24 hours at room temperature. Then, undissolved $N, N^{\prime}$-dicyclohexylurea was removed by filtration. The solution was precipitated in cold ethanol. The precipitation was collected by centrifugation and dissolved in water. The mixture solution was transferred to a dialysis bag $(\mathrm{MWCO}=14 \mathrm{kDa})$ and dialyzed against distilled water for 2 days. The white solid was obtained after freeze-dried (“P70d”, 0.92 g, yield, 94\%).

P70d with varying degree of substitution (6.8 and 10.5) were obtained by using different molar ratios of lipoic acid anhydrides to AHG of dextran (0.6 and 0.7).

Reduction Model reaction: The aim of the small molecule model reaction is to study the reduction of the lipoic acid dithiolane ring by DTT in PB8.2. The reaction of sodium lipoate and DTT was followed by ${ }^{1} \mathrm{H}$ NMR. To a solution of sodium lipoate $(5 \mathrm{mg}, 0.02 \mathrm{mmol})$ in PB8.2 $(450 \mu \mathrm{L})$ and 4 drops of $\mathrm{D}_{2} \mathrm{O}$, a solution of DTT $(3.4 \mathrm{mg}, 0.02 \mathrm{mmol})$ in PB8.2 $(500 \mu \mathrm{L})$ was added and the reaction was checked after 10 minutes by ${ }^{1} \mathrm{H}$ NMR at room temperature.

Preparation of Dex $\boldsymbol{P}-\boldsymbol{A}$ hydrogels: P70-4.2 (40 mg, containing $0.0104 \mathrm{mmol}$ lipoic acid group) was dissolved in $360 \mu \mathrm{L}$ phosphate buffer solution (100 mM, pH=8.2; 'PB8.2'). The mixture was stirred for 15 minutes at room temperature to dissolve Dex-P completely. Dithiothreitol (16 mg, 0.104 mmol, "DTT") was dissolved in $200 \mu \mathrm{L}$ PB8.2 as DTT pre-solution. 3-Butyn-2-one A $(8.2 \mu \mathrm{L}, 0.104 \mathrm{mmol})$ was added in $200 \mu \mathrm{L}$ PB8.2 as alkynone A pre-solution. $20 \mu \mathrm{L}$ DTT pre-solution $(0.0104 \mathrm{mmol})$ was added to the Dex-P solution and stirred for 10 minutes. Then $20 \mu \mathrm{L}$ A pre-solution $(0.0104 \mathrm{mmol})$ was added. A turbid hydrogel $(40 \mathrm{mg}$ 
polymer in $400 \mu \mathrm{L}, 10 \mathrm{wt} \%$ ) formed after 4 hours in a $1.5 \mathrm{~mL}$ glass vial at room temperature. Gelation was checked by the vial inversion method every half an hour.

P70 with varying DS (3.1 and 5.9), P500-4.8, P20-6.4 and P70d with varying DS (4.2, 6.8 and 10.5) were tested for gelation by the method described above.

Rheological measurements of hydrogels: A Dex P-A hydrogel was prepared as described above. A $500 \mu \mathrm{L}$ solution of pre-gel solution was positioned on the rheometer plate. Time sweep measurements were performed at fix strain $(\gamma=0.5 \%)$ and frequency $(\omega=6.28 \mathrm{rad} / \mathrm{s}=1 \mathrm{~Hz})$. Frequency sweep measurements were performed from 100 to $0.01 \mathrm{rad} / \mathrm{s}$ at fix strain $(\gamma=0.5 \%)$. All frequency sweeps were measured after storage modulus $\left(G^{\prime}\right)$ reached equilibrium state. All measurements were performed in the linear viscoelastic region. The modulus of hydrogels was measured under strain sweep from 1 to $1200 \%$ at a fixed frequency $(\omega=6.28 \mathrm{rad} / \mathrm{s})$. Continuous step strain measurements were measured at fixed frequency $(\omega=6.28 \mathrm{rad} / \mathrm{s})$. Oscillatory strains were switched from $1 \%$ strain to subsequent $1200 \%$ strain with 3 minutes for every strain period.

Self-healing test and injection of hydrogel: Two pieces of cube-shaped hydrogel $(4 \times 10 \times 10$ $\mathrm{mm}$ ) were prepared as described above colored by rhodamine B (red dye) and fluorescein (yellow dye). Then two piece of different color hydrogels were brought together and kept in a moist environment for 10 minutes. Afterwards, this healed hydrogel was cut to 4 equal pieces using a scalpel. Then a piece of hydrogels was put in a syringe ( $1 \mathrm{~mL}$ volume; 0.5 inner diameter) using a tweezer and syringe plunger, and subsequently injected through a $20 \mathrm{G}$ needle using manual force.

Cell viability assay: The cytotoxicity of the Dex P-A hydrogel was evaluated through an MTT (tetrazolium (3-(4,5-dimethylthiazol-2-yl)-2,5-diphenyltetrazolium bromide) assay, as previously described. ${ }^{[29]}$ Briefly, $85 \mu \mathrm{L}$ of Dex P-A hydrogel were added per well to a 96-well tissue-culture plate. The hydrogel was washed by adding $100 \mu \mathrm{L}$ of PBS to eluted unreacted reagents. Then, D1 cells (100 000 cells per well) were added in $150 \mu \mathrm{L}$ of IMDM complete medium. As a negative control of toxicity, D1 cells on IMDM complete medium were used. In addition, DMSO (5\%) was used as a positive control of toxicity. The cells were incubated with the Dex P-A hydrogel for 24 or $48 \mathrm{~h}$ at $37^{\circ} \mathrm{C}, 5 \% \mathrm{CO}_{2}$, and $95 \%$ humidity. Next, cells were spun down three minutes at 300xg at room temperature. Later, the medium was removed from each well and replaced with $90 \mu \mathrm{L}$ of IMDM complete medium. Then $10 \mu \mathrm{L}$ of MTT (final concentration of $0.5 \mathrm{mg} / \mathrm{ml}$ in PBS) was added to each well and incubated for $3 \mathrm{~h}$ at $37^{\circ} \mathrm{C}, 5 \%$ 
$\mathrm{CO}_{2}$, and $95 \%$ humidity. The formazan was precipitated by centrifugation, the medium was removed, and $100 \mu \mathrm{L}$ of DMSO was added to each well to dissolve the formazan crystals. The plate was incubated for $30 \mathrm{~min}$ at $37^{\circ} \mathrm{C}$, with $5 \% \mathrm{CO}_{2}$, and $95 \%$ humidity. Absorbance at $570 \mathrm{~nm}$ was measured using a CLARIOstar plate reader. The number of surviving cells is directly proportional to the amount of formazan product. Results were normalized between untreated cells as $100 \%$ and only media as background signal.

SIINFEKL release assay: To evaluate indirectly the release of SIINFEKL from the Dex P-A hydrogel a T-cell activation assay was carried out as previously described. ${ }^{[31]}$ Briefly, 1, 10, and $100 \mu \mathrm{M}$ of SIINFEKL peptide were added to Dex P-A hydrogel. Then $85 \mu \mathrm{L}$ of hydrogel was added per well to a 96-well tissue-culture plate. The hydrogel was washed with $100 \mu \mathrm{L}$ of PBS $1 \mathrm{x}$ and later incubated with $100 \mu \mathrm{L}$ of fresh IMDM medium for 2, 4, 6, 8, 10, 24, 34, and $48 \mathrm{~h}$ at $37^{\circ} \mathrm{C}, 5 \% \mathrm{CO}_{2}$, and $95 \%$ humidity. The supernatant removed from the Dex P-A hydrogel was diluted 1:100, 1:1000 and 1:10000 for the 1, 10, and $100 \mu \mathrm{M}$ of SIINFEKL loaded hydrogel, respectively. The D1 cells (50 000 cells/well) were seeded in a 96-well plate and allowed to adhere to the plate for $1 \mathrm{~h}$ at $37^{\circ} \mathrm{C}, 5 \% \mathrm{CO}_{2}$, and $95 \%$ humidity. The diluted supernatant was added to the D1 cells. The D1 cells were pulsed with $100 \mu \mathrm{L}$ of each supernatant timeslot for $3 \mathrm{~h}$ at $37^{\circ} \mathrm{C}, 5 \% \mathrm{CO}_{2}$, and $95 \%$ humidity. The cells were spin down at $300 \mathrm{xg}$ per $5 \mathrm{~min}$, the medium was removed, and $50000 \mathrm{~B} 3 \mathrm{Z}$ T-cells were added per well. Co-cultures were incubated overnight ( $15 \mathrm{~h}$ ) at $37^{\circ} \mathrm{C}, 5 \% \mathrm{CO}_{2}$, and $95 \%$ humidity.

The T-cell activation was measured as beta-galactosidase-directed CPRG (chlorophenol red- $\beta$ galactopyranoside) hydrolysis. The B3Z T-cell line carries a lacZ construct driven by NFAT, therefore the CPRG assay has a direct correlation with IL-2 promotor activity. For the CPRG assay, $100 \mu \mathrm{L}$ of lysis buffer were added per well and incubated for $4 \mathrm{~h}$ at $37^{\circ} \mathrm{C}$ in the dark. The absorbance was measured at $570 \mathrm{~nm}$ in a CLARIOstar plate reader.

For each assay, a standard curve was performed to interpolate the concentration of SIINFEKL available to pulsed dendritic cells and therefore activated the B3Z cells. In this regard, the data was interpolated into a curve of 5-0.1 nM of SIINFEKL peptide using a lineal regression.

Statistical analysis: Mean and Standard Error of Mean (SEM) and Standard Deviation (SD) were calculated for each variable studied. Based on the normality test, the difference between groups were assessed using a parametric test ( $t$ test). To interpolate the standard curve, we used a logarithmic regression. In all cases, a value of $\mathrm{p}<0.05$ was considered statistically significant. All 
graphics were performed using GraphPad Prism software, version 6.00 for MAC (GraphPad Software, La Jolla, California, USA, www.graphpad.com), and the statistical analyses was performed in R Studio (Version 0.98.1091 - (C) 2009-2014 RStudio, Inc.) using the package pracma.

\section{Acknowledgements}

Financial support by the Chinese Scholarship Council (B.F.) and the European Research Council (R.E., ERC consolidator grant 726381) is acknowledged.

\section{Conflict of Interest}

The authors declare no financial/commercial conflict of interest.

\section{References}

[1] D. J. Irvine, M. A. Swartz, G. L. Szeto, Nat. Mater. 2013, 12, 978.

[2] A. Lees, F. Finkelman, J. K. Inman, K. Witherspoon, P. Johnson, J. Kennedy, J. J. Mond, Vaccine 1994, 12, 1160.

[3] K. M. Cirelli, D. G. Carnathan, B. Nogal, J. T. Martin, O. L. Rodriguez, A. A. Upadhyay, C. A. Enemuo, E. H. Gebru, Y. Choe, F. Viviano, C. Nakao, M. G. Pauthner, S. Reiss, C. A. Cottrell, M. L. Smith, R. Bastidas, W. Gibson, A. N. Wolabaugh, M. B. Melo, B. Cossette, V. Kumar, N. B. Patel, T. Tokatlian, S. Menis, D. W. Kulp, D. R. Burton, B. Murrell, W. R. Schief, S. E. Bosinger, A. B. Ward, C. T. Watson, G. Silvestri, D. J. Irvine, S. Crotty, Cell 2019, 177, 1153.

[4] A. Bolhassani, S. Safaiyan, S. Rafati, Mol. Cancer 2011, 10, 3.

[5] D. T. O’Hagan, H. Jeffery, M. J. J. Roberts, J. P. McGee, S. S. Davis, Vaccine 1991, 9, 768.

[6] N. A. Peppas, P. Bures, W. Leobandung, H. Ichikawa, Eur. J. Pharm. Biopharm. 2000, $50,27$.

[7] S. Naahidi, M. Jafari, M. Logan, Y. Wang, Y. Yuan, H. Bae, B. Dixon, P. Chen, Biotechnol. Adv. 2017, 35, 530.

[8] L. García-Fernández, M. Olmeda-Lozano, L. Benito-Garzón, A. Pérez-Caballer, J. San Román, B. Vázquez-Lasa, Mater. Sci. Eng. C 2020, 110, 110702.

[9] T. Vermonden, R. Censi, W. E. Hennink, Chem. Rev. 2012, 112, 2853.

[10] P. B. O’Donnell, J. W. McGinity, Adv. Drug Deliv. Rev. 1997, $28,25$.

[11] P. Sansdrap, A. J. Moës, Int. J. Pharm. 1992, 98, 157.

[12] S. Pacelli, P. Paolicelli, M. A. Casadei, Carbohydr. Polym. 2015, 126, 208. 
[13] W. E. Hennink, C. F. van Nostrum, Adv. Drug Deliv. Rev. 2002, 54, 13.

[14] A. M. Kloxin, C. J. Kloxin, C. N. Bowman, K. S. Anseth, Adv. Mater. 2010, 22, 3484.

[15] G. A. Roth, E. C. Gale, M. Alcántara-Hernández, W. Luo, E. Axpe, R. Verma, Q. Yin, A. C. Yu, H. Lopez Hernandez, C. L. Maikawa, A. A. A. Smith, M. M. Davis, B. Pulendran, J. Idoyaga, E. A. Appel, ACS Cent. Sci. 2020, 6, 1800.

[16] S. D. Allison, J. Pharm. Sci. 2008, 97, 2022.

[17] R. Langer, J. Folkman, Nature 1976, 263, 797.

[18] C. J. M. Melief, T. van Hall, R. Arens, F. Ossendorp, S. H. van der Burg, J. Clin. Invest. 2015, 125,3401 .

[19] P. A. Ott, Z. Hu, D. B. Keskin, S. A. Shukla, J. Sun, D. J. Bozym, W. Zhang, A. Luoma, A. Giobbie-Hurder, L. Peter, C. Chen, O. Olive, T. A. Carter, S. Li, D. J. Lieb, T. Eisenhaure, E. Gjini, J. Stevens, W. J. Lane, I. Javeri, K. Nellaiappan, A. M. Salazar, H. Daley, M. Seaman, E. I. Buchbinder, C. H. Yoon, M. Harden, N. Lennon, S. Gabriel, S. J. Rodig, D. H. Barouch, J. C. Aster, G. Getz, K. Wucherpfennig, D. Neuberg, J. Ritz, E. S. Lander, E. F. Fritsch, N. Hacohen, C. J. Wu, Nature 2017, 547, 217.

[20] G. Sun, C. C. Chu, Carbohydr. Polym. 2006, 65, 273.

[21] S. Mitra, U. Gaur, P. C. Ghosh, A. N. Maitra, J. Control. Release 2001, 74, 317.

[22] S. R. Van Tomme, W. E. Hennink, Expert Rev. Med. Devices 2007, 4, 147.

[23] B. Fan, K. Zhang, Q. Liu, R. Eelkema, ACS Macro Lett. 2020, 9, 776.

[24] G. Joshi, E. V Anslyn, Org. Lett. 2012, 14, 4714.

[25] H.-Y. Shiu, T.-C. Chan, C.-M. Ho, Y. Liu, M.-K. Wong, C.-M. Che, Chem. Eur. J. 2009, 15,3839 .

[26] N. Van Herck, D. Maes, K. Unal, M. Guerre, J. M. Winne, F. E. Du Prez, Angew. Chem. Int. Ed. 2020, 59, 3609.

[27] T. Liebert, S. Hornig, S. Hesse, T. Heinze, J. Am. Chem. Soc. 2005, 127, 10484.

[28] C. Winzler, P. Rovere, M. Rescigno, F. Granucci, G. Penna, L. Adorini, V. S. Zimmermann, J. Davoust, P. Ricciardi-Castagnoli, J. Exp. Med. 1997, 185, 317.

[29] M. B. Hansen, S. E. Nielsen, K. Berg, J. Immunol. Methods 1989, 119, 203.

[30] O. Rötzschke, K. Falk, S. Stevanović, G. Jung, P. Walden, H. G. Rammensee, Eur. J. Immunol. 1991, 21, 2891.

[31] J. Karttunen, N. Shastri, Proc. Natl. Acad. Sci. U. S. A. 1991, 88, 3972.

[32] J. Long, A. V Nand, C. Bunt, A. Seyfoddin, Pharm. Dev. Technol. 2019, 24, 839.

[33] M. L. Bruschi, Ed. , in Strategies to Modify Drug Release from Pharmaceutical Systems, Woodhead Publishing, 2015, pp. 63-86.

[34] K. Adu-Berchie, D. J. Mooney, Acc. Chem. Res. 2020, 53, 1749.

[35] J. S. Moore, S. I. Stupp, Macromolecules 1990, 23, 65. 
[36] A. Sadownik, J. Stefely, S. L. Regen, J. Am. Chem. Soc. 1986, 108, 7789.

[37] Y.-L. Li, L. Zhu, Z. Liu, R. Cheng, F. Meng, J.-H. Cui, S.-J. Ji, Z. Zhong, Angew. Chem. Int. Ed. 2009, 48, 9914. 